\title{
Poster Session
}

\section{[Poster 1]}

\section{Generation of monoclonal antibodies directed} against peptide sequences by a $B$ cell targeting technique

M. Tomita ${ }^{\mathrm{a}, *}$, T. Taniguchi ${ }^{\mathrm{a}}$, J. Tanaka ${ }^{\mathrm{a}}$, H. Sawada ${ }^{\mathrm{b}}$, N. Nishino ${ }^{\mathrm{b}}$, T.Y. Tsong ${ }^{\mathrm{c}}$ and T. Yoshimura ${ }^{\mathrm{a}}$

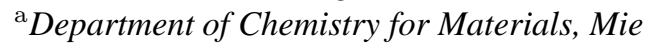
University, Tsu 514-8507, Japan

${ }^{\mathrm{b}}$ Graduate School of Life Science and Systems

Engineering, Kyushu Institute of Technology,

Kitakyushu 808-0196, Japan

${ }^{\mathrm{c}}$ Department of Biochemistry, University of

Minnesota, St. Paul, MN 55108-1022, USA

${ }^{*}$ Tel.: +8159231 9429; Fax: +81 592319430 ;

E-mail: tomita@chem.mie-u.ac.jp

Abstract: An in vitro system enabled us to accomplish the immunization in only 3 to 5 days, and also small amount of antigen with 1 to $10 \mu \mathrm{g}$ was enough for the immunization. We employed human insulin as an antigen for this purpose. Two putative epitope sequences of human insulin were selected on the basis of a BLAST analysis. Firstly, the targeting of B lymphocytes by these epitope sequences after in vitro immunization were immunofluorescently confirmed, and then the peptide-selected B lymphocytes were combined with myeloma cells by exploiting the specificity and strength of the interaction between biotin and avidin. Finally, these cell complexes were selectively fused by an electrical pulse. In consequence, several clones secreting monoclonal antibodies against each peptide sequence were successfully obtained, although they showed rather polyreactive specificities.

We suggest that the targeting technique have driven efficient production of monoclonal antibodies against the peptide sequences even after short immunization. This system would be applicable to generate hybridoma cells that can secrete human monoclonal antibodies which harbor relatively broad reactivities.

Introduction: A targeting technique, which is also known as a pulsed electric field (PEF) method, can realize selective production of monoclonal antibodies against the antigens of interest with high efficiency and selectivity. This new technique has enabled us to produce hybridoma cells secreting the desired monoclonal antibodies with much higher efficiency [1-4] than that obtained by a poly(ethylene glycol) (PEG) method [5,6]. Recently, we have succeeded in generating monoclonal antibodies directed against the aimed peptide sequences of presenilin 1 that is believed to be related to the early-onset of familial Alzheimer's disease [7].

In the present study, we have focused on further developing this technique using in vitro immunization system to obtain monoclonal antibodies in the short term.

Determinations of epitope sequences of human insulin based on a BLAST analysis: Figure 1 shows the primary structures of human and mouse insulins. Two putative epitope sequences of human insulin, termed B-1-II and B-3-II, were selected on the basis of a Basic Local Alignment Search Tool (BLAST) analysis. The selected peptide sequences showed no hitting with the primary structures of any mouse protein molecules, and those two peptide sequences preferentially crossreacted with anti-human insulin antibodies utilizing an enzyme-linked immunosorbent assay (ELISA) method.

In vitro immunization: A spleen from a mouse with 4 to 10 weeks was aseptically removed in a laminar flow hood. All cells containing lymphocytes were collected at $800 \mathrm{xg}$ for $5 \mathrm{~min}$. For the sensitization, $10 \mu \mathrm{g}$ of human insulin was added to a $10-\mathrm{ml}$ suspension of lymphocytes together with $20 \mu \mathrm{g} / \mathrm{ml}$ of muramyl dipeptide, N-acetylmuramyl-L-alanyl-D-isoglutamine, as adjuvant peptide. The $10-\mathrm{ml}$ suspension was placed into a $25-\mathrm{cm}^{2}$ tissue culture flask and incubated at $37^{\circ} \mathrm{C}$ in a humidified $5 \% \mathrm{CO}_{2} / 95 \%$ air atmosphere for 4 days prior to fusion (Fig. 2) [8]. In some experiments, IL-4 and LPS were included during immunization.

Immunofluorescent analysis of B lymphocytes: An immunofluorescent analysis was performed to clarify the selection of B lymphocytes targeted by the peptides of B-1-II and B-3-II. As shown in Fig. 3, $\mathrm{B}$ lymphocytes immunized in vitro by human insulin 
A鎖

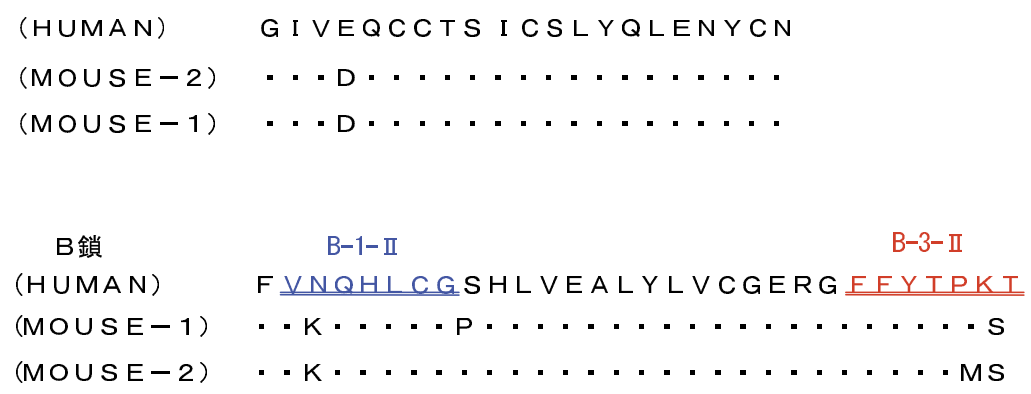

Fig. 1. Primary structures of human and mouse insulins. Two putative epitope sequences of human insulin that are B-1-II and B-3-II, were selected by a BLAST analysis.

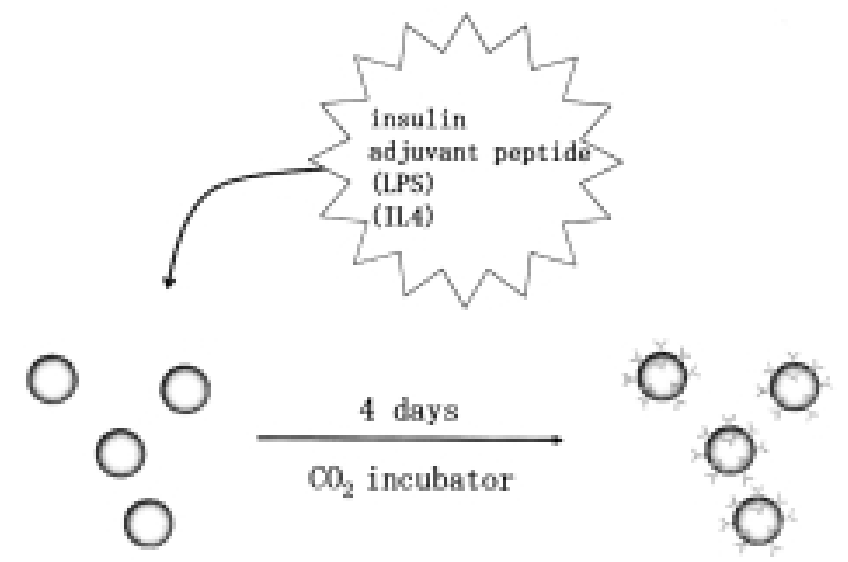

Fig. 2. In vitro immunization. An in vitro immunization was carried out in the presence of human insulin and muramyl dipeptide in a humidified $5 \% \mathrm{CO}_{2}$ incubator at $37^{\circ} \mathrm{C}$ for 4 days.

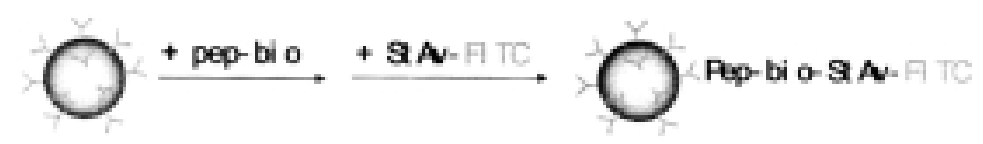

B cesls

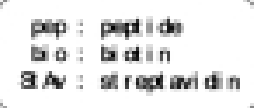

Fig. 3. Immunofluorescent analysis of B lymphocytes. B lymphocytes immunized in vitro were analyzed by an immunofluorescent method as follows: The B lymphocytes immunized by human insulin were selected by biotinylated B-1-II and B-3-II, followed by labeling with FITC-conjugated streptavidin.

were selected by biotinylated peptides, and labeled with streptavidin-FITC. The resulting B lymphocytepeptide-biotin-streptavidin-FITC complexes were analyzed by confocal laser microscopy. As a result, the B lymphocytes selected by B-1-II and B-3-II were successfully detected even after the short immunization. The B lymphocytes harboring antigen-specific recep- tors were generated and targeted by the aimed epitope sequences. In control experiments, B lymphocytes from a non-immunized mouse were employed instead of those of the immunized mouse, and the same peptide conjugates failed to recognize B lymphocytes from the non-immunized mouse.

B cell targeting technique: B lymphocytes im- 
munized by in vitro system were preselected by the peptides, B-1-II and B-3-II, based on the surface immunoglobulin receptors on B lymphocytes. The peptide-selected B lymphocytes were brought into contact with myeloma cells by harnessing the power of the specific and strong interactions between biotin and avidin. Finally, B lymphocytepeptide-biotin-streptavidin-biotin-myeloma cell complexes were fused by imposing an electric field of a few $\mathrm{kV} \mathrm{cm}{ }^{-1}$ by a high-voltage pulse machine for $10 \mu \mathrm{s}$, four times, generating an electrical pulse with a square wave.

Generation of hybridoma cells: Hybridoma cells were successfully yielded at the rate of approximately $10 \%$ by a B cell targeting technique after in vitro immunization. Among them, we were able to obtain several hybridoma cells secreting monoclonal antibodies against the peptides of interest, although they showed relatively broad cross-reactivities. This would be attributed to insufficient differentiation of B lymphocytes due to the short immunization. However, it is worth noting that we have found and succeeded in selecting the B lymphocytes expressing the immunoglobulin receptors on their cell surfaces even after the short immunization. Our preliminary results show that the yielding of hybridoma cells was much enhanced by addition of IL-4 and LPS during in vitro sensitization.

One particular advantage of this method is that targeting B lymphocytes can stringently isolate specific cells in advance, thereby leading to the selective production of monoclonal antibodies against the aimed peptide sequences.

\section{References}

[1] M.M.S. Lo, T.Y. Tsong, M.K. Conrad, S.M. Strittmatter, L.D. Hester and S.H. Snyder, Monoclonal antibody production by receptor-mediated electrically induced cell fusion, Nature $\mathbf{3 1 0}$ (1984), 792-794.

[2] T.Y. Tsong, M. Tomita and M.M.S. Lo, Pre-selection of Blymphocytes by antigen for fusion to myeloma cells by pulsed electric field (PEF) method, in: Molecular Mechanisms of Membrane Fusion, S. Ohki, D. Doyle, T.D. Flanagan, S.W. Hui and E. Mayhew, eds, Plenum, New York, 1988, pp. 223-236.

[3] M. Tomita and T.Y. Tsong, Selective production of hybridoma cells: antigenic-based pre-selection of B lymphocytes for electrofusion with myeloma cells, Biochim. Biophys. Acta $\mathbf{1 0 5 5}$ (1990), 199-206.

[4] T.Y. Tsong and M. Tomita, Selective B lymphocyte-myeloma cell fusion, Methods Enzymol. 220 (1993), 238-246.

[5] G. Köhler and C. Milstein, Continuous cultures of fused cells secreting antibody of predefined specificity, Nature 256 (1975), 495-497.

[6] S.F. De St. Groth and D. Scheidegger, Production of monoclonal antibodies: strategy and tactics, J. Immunol. Methods $\mathbf{3 5}$ (1980), 1-21.
[7] M. Tomita, H. Sugi, K. Ozawa, T.Y. Tsong and T. Yoshimura, Targeting antigen-specific receptors on B lymphocytes to generate high yields of specific monoclonal antibodies directed against biologically active lower antigenic peptides within presenilin 1, J. Immunol. Methods 251 (2001), 31-43.

[8] B.D. Boss, An improved in Vitro immunization procedure for the production of monoclonal antibodies, Methods Enzymol. 121 (1986), 27-33.

[Poster 2]

Production and optimization of ovine anti-tetanus antibody

El-Rashdy M. Redwan ${ }^{\mathrm{a}, *}$, Ahmed Khalil ${ }^{\mathrm{b}}$ and Zeinab Z. EL-Dardiri ${ }^{\mathrm{c}}$

${ }^{a}$ Protein Research Department, GEBRI Mubarak City for Scientific Research and Biotechnology

Application, Alexandria

${ }^{\mathrm{b}}$ Medical Biotechnology, GEBRI Mubarak City for

Scientific Research and Biotechnology Application, Alexandria

${ }^{\mathrm{c}}$ Biochemistry Department, Faculty of Science, Ain

Shams University, Cairo, Egypt

${ }^{*}$ Corresponding author.

E-mail: elrashdy@yahoo.com

Clostridium tetanus is a common soil organism. The organism expresses a potent neurotoxin of $150 \mathrm{kDa}$ which is responsible for much of the pathology associated with tetanus. To treat tetanus; there are both human and horse derived anti-tetanus products available commercially. The human product, tetanus immune globulin (TIG), is relatively safe, although, as with any human source biopbarmaceutical, there is a chance of passing blood borne pathogens. Unfortunately, the horse anti-tetanus and anti-venom products produce adverse reactions in 6-10\% of adult patients. Ovine serum has been chosen as the source of immunoglobulin for the successful cardiac glycoside treatment, Digibind ovine Fab. The use of Digibind in the USA has been estimated at about 2500-treatment/year. Experience with Digibind has shown an overall incidence of adverse reactions of $0.8 \%$ and no anaphylaxis has been reported. In addition, sheep are relatively inexpensive, widely available specifically in developing countries, and rapidly achieve high circulating levels of highaffinity IgG antibodies. We plan to use the ovine as host to production and optimization of anti-TT antibody. Four female sheep were immunized with human vaccine (TT-Alum) every two weeks for 16 weeks, serum was collected and its titer was estimated by ELISA. The highest titer we obtained was 39,000. To optimize a purification protocol for ovine anti-tetanus, we used four methods; weak anion (DEAE-), weak cation (CM-), 
ammonium sulfate precipitation $(20 \%, 30 \%, 40 \%$, and $50 \%$ ) alone or in combined with caprylic acid. Ammonium sulfate precipitation at $50 \%$ in combined with caprylic acid gave us the highest: yield, specific activity, purer Fab product. The figures and tables of our results will be shown.

[Poster 3]

Production of monoclonal antibody against human Epsilon $(\varepsilon)$ Chain

J. Majidi $^{\text {a }}$, A. Zavaran Hosseini ${ }^{\text {b }}$, Z.M. Hassan ${ }^{\mathrm{b}}$ and

M.H. Alimohammadian ${ }^{\mathrm{c}}$

${ }^{a}$ Department of Immunology, Faculty of Medicine, Tabriz University of Medical Science, Country?

E-mail: majidij@tbzmed.ac.ir

${ }^{\mathrm{b}}$ Department of Immunology, Faculty of Medicine,

Tarbiat Modarres University, Country?

${ }^{\mathrm{c}}$ Department of Immunology, Pasteur Institute of Iran, Iran

Epsilon $(\varepsilon)$ Chain is heavy chain of one of the five classes of immunglobulins that plays an important role in allergic diseases. Production of monoclonal antibodies by a single clonotype against different epitopes of Epsilon $(\varepsilon)$ Chain has high priority in development of diagnostic kits. In this study, an attempt was made to produce monoclonal antibodies against human Epsilon $(\varepsilon)$ Chain. Balb/c mice were immunized with semi purified Epsilon $(\varepsilon)$ Chain and spleen cells fused with SP2/0 mouse myeloma cell line in the presence of PEG (Poly Ethylene Glycol). Supernatant of hybridoma cells was screened for detection of antibody by ELISA method. Cloning of selective high absorbance wells were done with limiting dilution method. The suitable clone (monoclone) was selected by ELISA and confirmed by immunoblot. The subclass of the chosen monoclone antibodies was determined and the clones freezed and kept in liquid nitrogen. During this study three successful fusions were carried out, which resulted in the development of 156 clones with high production of anti-IgE.

Fourteen clones with the highest titers were selected for cloning. After limiting dilution more than 100 monoclonal antibodies were produced and the unsuitable one was $\mathrm{C} 1 \mathrm{~F} 2$, i.e.; the clone that displayed the high absorbance in reaction with purified IgE, relatively high cross-reactivity with $\operatorname{IgM}$, and the highest cross-reactivity with IgG. In immunoblotting, presence of relatively high-density band in reaction with $\operatorname{IgE}$ was confirmed. The unsuitable monoclonal antibody was shown to be IgG1 subclass with kappa light chain.
It seems that, this monoclonal antibody could not be successfully useful in diagnostic kits.

[Poster 4]

Chimeric anti-N-glycolyl-ganglioside and its anti-idiotype Mabs: Immunodominance of their variable region

Alejandro López-Requena, Cristina Mateo De Acosta, Alexis Pérez, Aisel Valle, Josefa Lombardero, Katya

Sosa, Rolando Pérez and Ana María Vázquez

Department of Antibody Engineering, Center of

Molecular Immunology, P.O. Box 16040, Havana 11600, Cuba

P3 $\mathrm{mAb}$ is a murine IgM that specifically recognizes $\mathrm{N}$-glycolyl (NeuGc)-gangliosides and sulfatides. It also reacts with antigens expressed in human breast tumors and melanoma. In syngeneic model, P3 mAb is able to elicit a strong anti-idiotypic (Ab2) antibody response, even in absence of adjuvants or carrier proteins. $1 \mathrm{E} 10 \mathrm{mAb}$ is an anti-idiotypic antibody specific for P3 $\mathrm{mAb}$ that has demonstrated anti-tumoral effects in syngeneic and allogeneic animals. Here we reported the construction of the human IgG1 chimeric P3 and 1E10 antibodies, and the evaluation of the maintenance of the main properties of the murine mAbs. Chimeric P3 antibody specifically reacted with GM3(NeuGc) and GM2(NeuGc) gangliosides, and not with their acetylated variants. Also, it strongly recognized the antiidiotypic 1E10 mAb. Chimeric 1E10 antibody specifically reacted with $\mathrm{P} 3 \mathrm{mAb}$. Upon immunization of Balb/c mice with both chimeric antibodies, we were able to demonstrate the immunodominance of their variable region. The anti-idiotypic response induced by both antibodies was very strong and in most of the mice was even significantly higher than the antiisotypic response, despite the fact that $70 \%$ of the chimeric molecule is xenogenic with respect to the animal model.

\section{[Poster 5]}

Incidence of human immunodeficiency virus antibodies among commercial blood donors in University of Benin Teaching Hospital (UBTH) F.E. Oronsaye

University of Benin, Edo State, Nigeria

Abstract not received. 
[Poster 6]

Use of the antigenic determinant recognized by human monoclonal antibody as a novel molecular target for cancer therapy

Yasuyuki Aotsuka, Junzo Nagao, Junichi Miyahara, Yasushi Yamamoto, Yuko Mitoh and Hideaki Hagiwara

Hagiwara Institute of Health, Kasai, Japan

Human monoclonal antibodies (HuMoAbs) are an attractive agent for cancer therapy due to their long halflife in blood and weak adverse effects. Furthermore, HuMoAbs are effective tools for discovering and validating molecular targets that may lead to the alteration of cancer phenotypes such as unlimited proliferation, metastasis, angiogenesis and evasion of apoptosis.

As a result of cell fusion using lymphocytes of cancer patients we have generated various human monoclonal antibodies some of which inhibit the growth of human cancer xenografts in nude mice. Analysis of the antigenic determinant that is recognized by antibodies with anti-cancer activity demonstrated that these antibodies bind to an epitope designated TA226. This epitope has an alpha-helical structure and resides in vimentin coil 2A-2B and alpha-actinin 4 spectrin repeats. Whereas, vimentin is a type III intermediate filament protein that is found in a wide range of cells of mesenchymal origin as well as undifferentiated cells, alpha-actinin 4 is an actin-bundling protein that is thought to be associated with cell motility and cancer invasion.

Although the mechanisms underlying the inhibitory effects of these antibodies on the proliferation of cancer cells remain unclear, since aforementioned proteins are known to be localized in the cytoplasm or nucleus but not on the surface of tumor cells, TA226 may be used as a therapeutical target and HuMoAbs against TA226 are suggested to be of clinical value for use as antitumor drugs.

\section{[Poster 7]}

Characterization of monoclonal antibodies against fragments of the Plasmodium falciparum merozoite surface protein one (MSP-1)

W.H. Leung, J. Choi, K.T. Yan, S.H. Yuen, C.S. Yin and W.K.K. Ho

Department of Biochemistry, The Chinese University of Hong Kong, Shatin, Hong Kong, China

The 42-kDa fragment (PfMSP-1 ${ }_{42}$ ) of the Plasmodium falciparum merozoite surface protein 1 (PfMSP-1) is one of the most promising vaccine candidates against the blood stage malarial parasite. Previous studies have shown that recombinant PfMSP-1 ${ }_{42}$ (Bvp42) produced in the baculovirus silkworm expression system had a correct conformation similar to the native PfMSP-1, which was able to elicit both in vitro and in vivo protective immunity against the parasite. The major problem in producing this recombinant protein is the production yield as the purification process is still under optimization and not satisfactory up to the moment. Recently, we highly expressed the $42 \mathrm{kDa}$ fragment in a novel $\mathrm{E}$. coli strain, Origami (DE3), which has mutations in the glutathione and thioredoxin reductase genes. Recombinant PfMSP- $1_{42}$ was expressed in high-level as a fusion protein mainly in inclusion body form. Complete solubilization of the inclusion bodies was achieved using a non-denaturing solubilizing buffer. The protein was purified to about $95 \%$ purity by $\mathrm{Ni}^{+}$-chelating chromatography following by DEAE ion-exchange chromatography. The final yield was about $40 \mathrm{mg} / \mathrm{L}$ bacterial culture. In order to verify the structure of this bacterial protein with the well characterized one expressed by baculovirus, we have generated two monoclonal antibodies (Mabs) against Bvp42. One of the Mabs recognized the disulfide bond sensitive epitope of Bvp42 while the other was reactive against both the native and reduced form. These Mabs were found to cross-react with the bacterial expressed fusion protein indicating the structural similarity between the two recombinant proteins. Using the same E. coli expression system, we have also successfully prepared a fusion protein of the 33-kDa fragment of PfMSP-1 ${ }_{42}$. This fragment is believed to contain $T$ cell epitopes that can enhance the inhibitory immunity of PfMSP-142 against the parasite. In order to evaluate this hypothesis, we h ave also prepared Mabs against the $33 \mathrm{kDa}$ fragment fusion protein. It is hoped that these Mabs could be used as tools to delineate the role of the $33 \mathrm{kDa}$ and $19 \mathrm{kDa}$ fragments in the elicitation of protective immunity against malaria.

\section{[Poster 8]}

Generation of human-mouse hybridomas secreting food allergen specific human monoclonal IgM antibodies

Hiroshi Shinmoto $^{\mathrm{a}}$, Shihoko Doi ${ }^{\mathrm{b}}$, Kenji Yamagishi ${ }^{\mathrm{b}}$, Toshiyuki Kimura ${ }^{\mathrm{b}}$, Masahiro Suzuki ${ }^{\mathrm{b}}$, Toshihiro

Takahashi $^{\mathrm{c}}$ and Michie Shimmoto ${ }^{\mathrm{d}}$

${ }^{a}$ National Food Research Institute, 2-1-12 Kannondai, Tsukuba 305-8642, Japan

E-mail: shinmoto@affrc.go.jp 
${ }^{\mathrm{b}}$ National Agricultural Research Center for Tohoku Region, 50 Harajuku-minami, Arai, Fukushima 960-2156, Japan

${ }^{\mathrm{c}}$ Faculty of Agriculture, Miyazaki University, 1-1 Gakuen-konohanadai-nishi, Miyazaki 889-2192, Japan

${ }^{\mathrm{d}}$ The Tokyo Metropolitan Institute of Medical Science, 3-18-22 Hon-komagome, Bunkyo-ku, Tokyo 113-8613, Japan

To analyze structure of food allergens, we planned to obtain human monoclonal antibodies to food allergens. Our method employed the transformation of human B-cells with Epstein-Barr virus (EBV) followed by cell fusion with mouse myeloma cells to establish stable human-mouse hybridomas. We obtained over 5,000 EBV transformed human B-cells secreting antibodies against rice, soybean, peanut, wheat, pork skin gelatin, milk proteins, egg-white proteins, and cedar pollen extracts. Human-mouse hybridomas secreting anti-rice and anti-peanut monoclonal antibodies were generated by cell fusion of transformed B-cells with mouse myeloma cells and epitope sequences of a rice allergen and a peanut allergen were analyzed.

Introduction: Mouse monoclonal antibodies and sera from patients allergic to certain food have been used to analyze antigenic determinants of food allergens $[4-6,8]$. However, mouse monoclonal antibodies and sera from patients may recognize different antigenic determinants. Furthermore, it seems very difficult to collect a sufficient amount of sera from patients of food allergies. For these reasons, it seemed to be useful to establish human B-cell lines secreting antibodies specific to food allergens.

Epstein-Barr virus (EBV) is a herpes virus that infects human B-cells to cause infectious mononucleosis. It is also known that EBV transforms and immortalizes human B-cells in vitro [7]. Those immortalized cells (B-lymphoblastoid cells, BLCs) secrete antibodies. In this study, we prepare an immortalized B-cell library stock as a source of antibodies and antibody-producing cells, and generate human-mouse hybridomas for an epitope analysis of food allergens.

Immortalization of human B-cells: We planned to transform more than 500,000 B-cell clones. We estimated that one B-cell out of 1,000 peripheral blood lymphocytes (PBLs) would be immortalized by EBV. A stock would contain 100 clones of immortalized BLCs, because our multi-clone immortalized B-cell library stock was from 100,000 PBLs.

PBLs were prepared from thirteen healthy donors. PBLs infected with EBV were seeded into 96 well micro-culture plates. After 2 to 3 weeks of culturing, wells with transformed lymphocytes were transferred to 24 well plates and cultured. Cells were further cultured in $6 \mathrm{~cm}$ dishes, and resulting BLCs were stored in liquid nitrogen. We obtained a total of 5,000 multiclone stocks of BLCs for the cell library. We estimated that our immortalized B-cell library might contain more than 500,000 clones of BLCs [12].

ELISA of the culture supernatants: Culture supernatants of BLCs were analyzed for antibodies to several food allergens by enzyme linked immunosorbent assay (ELISA). Crude extracts from rice, soybean, two hen egg allergens (ovalbumin and ovomucoid), two cow milk allergens (alpha-s1-casein and beta-lactoglobulin), and ceder pollen allergens were used. We detected many cells secreting antibodies to food allergens. This result suggested that we obtained numerous BLCs secreting specific antibodies to many antigens.

Antibodies of $\operatorname{IgM}$ class were dominant. EBV is known to bind CR2 molecule (CD21, a $145 \mathrm{kDa}$ complement receptor) on B-cells of peripheral blood that express IgM class antibodies on the cell surface [2]. That would be the reason for dominant secretion of IgM by BLCs from PBLs [11].

Estimation of antigenicity of collagenase-treated gelatin with human antibodies secreted by BLCs: Gelatin has been used as a pharmaceutical excipient (stabilizer or additive). While gelatin had low antigenicity, some patients were found to be allergic to gelatin that was added to parmaceuticals. To decrease the antigenicity of gelatin, gelatin was digested by limited collagenase treatment and low allergic gelatins (FreAlagin) were developed [9].

Culture supernatants of BLCs were assayed for antibodies to porcine skin gelatin by ELISA. We obtained 74 BLC stocks containing cells secreting gelatin specific IgM class antibodies. Four of them (2-223, 3-192, 4-232, and 5-60) were chosen for a competitive ELISA.

Three collagenase-treated gelatins (R, R(2), and AD) had only weak inhibition of binding of antibodies 2-223 and 5-60. Binding of antibody 4-232 to the plate was not only inhibited with intact gelatin but also with high concentrations of enzyme-treated gelatins. Antibody 3-192 was not inhibited by any specimens.

Competitive ELISA of three types of collagenasetreated gelatins with antibodies 2-223 and 5-60 showed that three gelatin specimens could not inhibit the binding of antibodies to porcine skin gelatin. Antibodybinding sites on the gelatin molecules might be modified (or destroyed) by the treatment with collagenase. 
Those antibodies (2-223 and 5-60) could be useful to estimate antigenicity of collagenase-treated gelatin in an industrial process.

We believe that antibodies from immortalized human B-cells could be alternative reagents for analyzing antigenicity (or allergenicity) of gelatins treated with collagenase.

Rice allergen specific human monoclonal antibodies: BLCs secreting antibodies to rice allergens were fused with 8-azaguanine and ouabain resistant mouse myeloma SP2/O3 cells and the fused cells were cultured in a HAT medium containing ouabain. We obtained five stable human-mouse hybridomas secreting human monoclonal IgM class antibodies to rice allergens.

Western blot analysis of rice allergens with five human-mouse hybridomas showed four human monoclonal antibodies reacted with rice proteins having molecular weights of $14-16 \mathrm{kDa}$ and $25 \mathrm{kDa}$. One antibody, 3-207-2-6, reacted only with 14-16 kDa allergens. Proteins of $14-16 \mathrm{kDa}$ are thought to be rice major allergens reported by Alvarez et al. [1]. These proteins were reported to include several proteins with similar molecular weight and primary structures.

We chose one major allergen, RA17, for the epitope analysis. To detect the binding sequence of the RA17 allergen, we synthesized series of peptide (overlapping peptides) on a multi-pin apparatus. We prepared 63 deca peptide (10 amino acids) by repeating the shift of every two peptides in the direction from $\mathrm{N}$ to $\mathrm{C}$ terminus of the sequence of the RA17 allergen. Monoclonal antibody from human-mouse hybridoma 5-1757-2 was reacted with the pins. The strongest binding of the monoclonal antibody was on the Pins \# 59-61; near the C-terminus region of the RA17 allergen with a sequence of GPGGVCYWLGYPRT.

Peanut allergen specific human monoclonal antibodies: We also obtained five hybridomas secreting human monoclonal antibodies to peanut allergens. Five human monoclonal antibodies reacted with peanut proteins. Antibody 1 reacted with a protein with high molecular weight, and antibody 2 produced smear staining on the transferred membrane. Antibodies 3, 4, and 5 exhibited stained bands with 40 to $45 \mathrm{kDa}$ band; molecular weights were similar to that of Ara h1 [3,10]. Results of Western blot analysis of purified Ara h1 with five human monoclonal antibodies showed that antibody 4 and antibody 5 reacted with Ara h1. Antibody 2 produced smear staining on the Ara h1 transferred membrane.

To detect the binding sequences in Ara h1 allergen, we synthesized overlapping peptides. We prepared peptides ( 20 amino acids) by repeating the shift of every ten amino acids in the direction from $\mathrm{N}$ to $\mathrm{C}$ terminus of the sequence of Ara h1 protein. Monoclonal antibodies from human-mouse hybridomas 4 and 5 were reacted with the pins. The antibody 4 reacted with Pin \#5 with an amino acid sequence of RCLQSCQQEPDDLKQKACES, and antibody 5 reacted with Pin \#45 with a sequence of KAMVIVVVNKGTGNLELVAV. The sequence of Pin \#5 included a 48-57 sequence QEPDDLKQKA that was reported to be one of the Ara h1 epitopes determined by using sera from patients allergic to peanut $[3,10]$. Pin \#45 did not contain any known epitope sequence of Ara h1 protein.

Conclusion: A B-cell library consisting of more than 5,000 stocks of BLCs could be an excellent source of antibodies to a wide spectrum of allergens. It seemed to contain antibodies to various food allergens. The disadvantages of this method lie in the instability of the BLC clones secreting antibodies of interest and, thus, in the difficulty in establishing single clones. In contrast, cell fusion of human lymphocytes (not transformed) with mouse myeloma cells yields high fusion efficiency and enables cloning by limiting dilution. However, it is difficult to establish human-mouse hybridomas secreting antibodies with a certain specificity. The method described in this paper combined the advantages of both the cell library and cell fusion with mouse myeloma, and eliminates disadvantages.

The presence of clones secreting antibodies to rice and peanut allergens from normal donors suggested that the library stocks contained antibodies to most food allergens.

\section{References}

[1] A.M. Alvarez, E. Fukuhara, M. Nakase, T. Adachi, N. Aoki, R. Nakamura and T. Matsuda, Biosci. Biotech. Biochem. 59 (1995), 1304-1308.

[2] N.A. Brown and G. Miller, J. Immunol. 128 (1982), 24-29.

[3] A.W. Burks, D. Shin, G. Cockrell, J.S. Stanley, R.M. Helm and G.A. Bannon, Eur. J. Biochem. 245 (1997), 334-339.

[4] A.W. Jr Burks, J.R. Brooks and H.A. Sampson, J Allergy Clin. Immunol. 81 (1988), 1135-1142.

[5] R. Gonzalez, F. Polo, L. Lapatero, F. Caravaca and J. Carreira, Clin. Exp. Allergy 22 (1992), 748-755.

[6] S. Kaminogawa, A. Enomoto, J. Kurisaki and K. Yamauchi, J. Biochem. 98 (1985), 1027-1032.

[7] D. Kozbor and J.C. Roder, J. Immunol. 127 (1981), 12751280.

[8] A. Plebani, P. Restani, A. Naselli, C.L. Galli, A. Meini, G. Cavagni, A.G. Ugazio and C. Poiesi, Clin. Exp. Allergy 27 (1997), 949-956.

[9] Y. Sakai, R. Yamato, M. Onuma, T. Kikuta, M. Watanabe and T. Nakayama, Biol. Pharm. Bull. 21 (1998), 330-334.

[10] D.S. Shin, C.M. Compadre, S.J. Maleki, R.A. Kopper, H. Sampson, S.K. Huang, A.W. Burks and G.A. Bannon, J. Biol. Chem. 273 (1998), 13753-13759. 
Table 1

Positivity with Histiotype of lesions

\begin{tabular}{lccc}
\hline Lesions & $\begin{array}{c}\text { Number of } \\
\text { cases tested }\end{array}$ & $\begin{array}{c}\text { Number of } \\
\text { positive cases }\end{array}$ & $\begin{array}{c}\text { Percentage } \\
\text { positivity }\end{array}$ \\
\hline (A) Benign & 4 & & \\
a) Juvenile polyps & 2 & 2 & 50 \\
b) Naevus & 4 & 3 & 50 \\
c) Pemfigus & & & 75 \\
(B) Premalignant & 4 & 3 & \\
a) Leukoplakia & 3 & 1 & 75 \\
b) Submucous fibrosis & 4 & 3 & 33 \\
c) Dysplasia & 1 & 1 & 75 \\
d) Adenomatous polyps & 3 & 1 & 100 \\
e) Adenofibrosis & & & 33 \\
(C) Malignant & 25 & 22 & 88 \\
a) Squamous cell carcinoma & 2 & 2 & 100 \\
b) Verruca carcinoma & 8 & 5 & 62.5 \\
c) Anaplastic carcinoma & 2 & 17 & 94.4 \\
d) Adeno carcinoma & 2 & 100 \\
e) Paget's disease of breast & & & \\
\hline
\end{tabular}

[11] H. Shinmoto, S. Dosako and S. Tanaka, Tokai J. Exp. Clin. Med. 17 (1992), 129-132.

[12] H. Shinmoto, K. Nakahara, M. Kobori and T. Tsushida, Biotechnology Techniques 12 (1998), 545-547.

\section{[Poster 9]}

\section{Immunohistochemical study of P21- ras an oncogene product on human tissues \\ V. Mehrotra ${ }^{\mathrm{a}}$ and V.L. Lahiri \\ ${ }^{a}$ Department of Biochemistry, HIMS, Dehradun and \\ S.N. Medical College, Agra, India \\ Tel.: +91 1352412032 ; \\ E-mail: mehrotravinit@redifmail.com}

Introduction: Oncogenes can activate as gene amplification, translocation, mutation and retroviral promoter/ enhancer insertion. The human c-myc oncogenes and its oncogenes product are implicated in cell proliferation and differentiation and appear to play an important role in cell cycle control [1-3]. Moreover, c-myc appears to immortalize some cells and to act in cooperation with another prominent oncogenes, ras that can transform immortalized cells through multi-step carcinogenesis [4]. This ras family, which has been identified as a normal cellular component, consists of three members, Ha-ras, Ki-ras and N-ras. They code for a 21,000-kilo Dalton protein designated P21, which is located on the inner surface of the plasma membrane and appears to transduce receptor-mediated external signals entering the cell by binding guanosine triphosphate (GPT). These genes are known to be activated by single point mutations and cause changes at amino acid position 12, 13 or 61 in P21 in some human solid tumours or acute myeloid leukemia [5-7]. They also involve over expression of the normal protooncogenes product. Increased P21 expression has been observed in a variety of human cancers including those of urinary bladder, colon, stomach, lung, prostate, lever, thyroid and breast in about $50 \%$ of the tested tumours [8-10]. However, from recent immunohistochemical studies it is evident that P21 expression in a given tumour, irrespective of its primary origin or stage may be heterogeneous; some tumours cells have no detectable P21, whereas others show a strongly positive reaction [11]. Based on the suggestions that ras genes and their products may be involved in early stages of carcinogenesis, we have studied the distribution of ras-oncogene (P21) in human epithelial cancers and precancerous lesions of all the grades, obtain as biopsy or autopsy with confirmed histological examinations, by the immunohistochemical technique.

Material and methods: Tissues: 3-5 $\mu \mathrm{m}$ cryostat sections of unpreserved fresh tissues from human epithelial cancers, precancerous lesions and normal from various anatomical sites were prepared. Remaining tissue was processed for paraffin sections and histomorphological diagnosis.

Anti-P21 ras $\mathrm{Ki} / \mathrm{Ha}$ affinity purified antibody was obtained from Triton Biosciences Inc, CA, USA. This antibody recognizes the human Ki-ras, Ha-ras and Nras oncogenes product and react with $\mathrm{P} 21$ in the tissues.

Immunohistochemical staining: A Peroxidase-antiPeroxidase method was used to demonstrate the protein in tissue sections [12]. The overall intensity of the reaction in the tissue studied was arbitrarily scored as $0,+,++$, or +++ .

Results: A positive reaction with primary antibody, indicating presence of the ras gene product $\mathrm{P} 21$ was 
seen as brown granular deposits throughout the cytoplasm. This is consistent with the inner membrane localization of the P21 molecule.

Table 1 indicated the percentage positivity with the histiotype of lesions. In the control group of morphologically normal /non-cancer tissue only occasional cell (other than parietal cells) exhibited a positive reaction. Staining intensity was usually weak to moderate, the mean percentage of cells positively stained for ras P21, irrespective of intensity was only $25 \%$.

In carcinomas the pattern of positive staining of the tumour cells ranged from very weak to very strong. There was one case of diffuse type carcinomas in which no reaction took place with the antibody. Within the remaining tumours the staining intensity varied from cell to cell in each specimen there were some morphologically malignant cells that failed to stain for ras $\mathrm{P} 21$. The mean percentage of positively stained cells in cancers was $90 \%$. These values were significantly higher than in control normal, benign and premalignant lesions.

Discussion: The oncogenes most frequently detected in human tumours belong to the ras gene family (Ha-ras, Ki-ras and N-ras) An immunohistochemical study or P21 - ras expression was carried out on benign, premalignant and malignant lesions using monoclonal antibody to P21. The P21 expression was significantly higher in cancer cell than control specimens [13]. Enhanced expression of ras gene product was demonstrated in certain human cancers using immunohistochemical techniques or western blot analysis. These findings suggest that an increased amount of ras gene product may alter the regulatory control of cell transformation [14]. In this context, the hypothesis is that normal ras protein binds GTP in response to some receptor-mediated signal and futher transduce the signal into the cell. Signal transmission would thus terminate following conversion of GTP to GDP by intrinsic GTPase activity. In mutated ras protein, GTPase activity is reduced, resulting in prolonged binding of GTP and prolonged activation. The latter contributed significantly to uncontrolled growth. It might also be possible that even without mutation, an intense increase in ras protein may result in prolonged GTP binding and hence prolonged activation, contributing to uncontrolled growth like that seen in various types of malignancies/ carcinomas. While normal/ control were unstained, the expression of the ras gene product P21 was observed in only $25-50 \%$ cases.

Thus P21 ras oncogenes is suggested as a GTP binding, apart of signal transducting systems and are present at the inner surface of plasma membrane. This oncogene provides an excellent class of tumour marker for targeting and therapy, using immunological, pharmacological or radiolabelled agents to inhibit their functioning in cancers.

\section{References}

[1] C. Brooksbank, Ras the magician (news), Nat Rev Cancer 2(4) (2002), 249.

[2] T.Y. Shih and M.O. Weeks, Oncogenes and cancer: the p21 ras genes, Cancer Invest 2 (1984), 109-123.

[3] P.H. Hamlyn and K. Sikora, Oncogenes, Lancet 2 (1983), 326329.

[4] A. Burgess, Growth factors and oncogenes, Immunology Today 6 (1985), 107.

[5] G.M. Cooper, Cellular transforming genes, Science 717 (1982), 801-806.

[6] R.W. Sweet, S. Yokoyama, T. Kamatta, J.R. Feramisco, M. Rosenberg and M. Gross, The product of ras is a GTPase and the T24 oncogenic mutant is deficient in this activity, Nature 311 (1984), 273-275.

[7] M. Barbacid, ras Genes, Ann Rev Biochem 56 (1987), 779-827.

[8] S. Pulciani, E. Santos, A.V. Lauver, L.K. Long, S.A. Aaranson and M. Barbacid, Oncogenes in solid human tumors, Nature 300 (1982), 539-542.

[9] M.E. Furth, T.H. Aldrich and C. Cordon-Cardo, Expression of ras protooncogene proteins in normal human tissues, Oncogene 1 (1987), 47-58.

[10] P.G. Chesa, W.J. Rettig, M.R. Melamed, L.J. Old and H.L. Niman, Expression of p21-ras in normal and malignant human tissues: Lack of association with proliferation and malignancy, Proc Natl Acad Sci USA 84 (1987), 3234-3238.

[11] D.I. Venter, S. Kumar, N.L. Tuzi and W.J. Gullick, Overexpression of the c-erb-2 oncoprotein in human breast carcinomas: Immunohistological assessment correlates with gene amplification, Lancet 2 (1987), 69-72.

[12] S.M. Hsu, L. Raine and H. Fanger, Use of avidin-biotinperoxidase complex $(\mathrm{ABC})$ in immunoperoxidase techniques: A comparison between $\mathrm{ABC}$ and unlabled Antibody (PAP) procedures, J Histochem Cytochem 29 (1981), 577-580.

[13] N. Ohuchi, A. Thor, D.L. Page, P.H. Hand, S.A. Halter and L. Schlom, Expression of the 21,000 molecular weight ras protein in a spectrum of benign and malignant human mammary tissues, Cancer Res 46 (1986), 2511-2519.

[14] L.B. Johannes, V. V-de Matty, J.E. Alex, W.G.J. Johanns, D. Rund, L. Bob and P.C. Loes, Mutation in N-ras predominates in acute myeloid leukemia, Blood 69 (1987), 1237-1241.

\section{[Poster 10]}

\section{Single chain antibody vaccination against human} ovarian cancer enhanced by microspheres and cytokines

Jie Ma and Zheng Li

State Key Laboratory of Molecular Oncology, Cancer Institute, Chinese Academy of Medical Sciences, PUMC, Panjiayuan, Beijing 100021, China

Experimental studies using monoclonal antibody have demonstrated that anti-idiotypic immunity can be 
enhanced by microspheres or cytokines. The underlying physiological principles behind these strategies involve sustained release of antigen and arousal of the immune system. In this study, a murine model was used to investigate the combination effect of microspheres and cytokines on anti-idiotypic response. A single chain antibody $\mathrm{CS} 10$, which recognizes the human ovarian cancer antigen CA125 was formulated in microspheres and injected to mice alone or in the presence of colony stimulating factor (GM-CSF) or tumor necrosis factor $(\mathrm{TNF}-\alpha)$. The immunization of mice with formulated single chain antibody and cytokines resulted in enhanced production of anti-idiotypic antibody, and which subsequently induced the production of anti-anti-idiotypic antibody. These results raise the possibility of cancer immunotherapy by administration of single chain antibody encapsulated in microspheres with GM-CSF or TNF- $\alpha$.

\section{[Poster 11]}

Reactivity and consequences of cell-surface recognition by germline-encoded polyreactive antibodies

Roopali Gandhi ${ }^{\mathrm{a}}$, Joy Das ${ }^{\mathrm{a}}$, Rohini Handa ${ }^{\mathrm{b}}$ and Rahul $\mathrm{Pal}^{\mathrm{a}}$

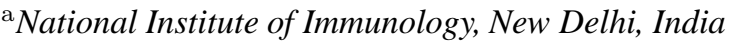

${ }^{a}$ All India Institute of Medical Sciences, New Delhi, India

Systemic lupus erythematosus (SLE) is non-organ specific, prototypic autoimmune disease, characterized by the presence of a range of antibodies against self-antigens. Auto-antibodies may contribute towards pathogenesis; for example, antibodies reactive towards double stranded (ds) DNA are associated with glomerulonephritis, and anti-Ro and/or anti-La antibodies may play pathogenic roles in clinical conditions like skin lesions and neonatal heart block. We investigated the consequences of autoimmune cell surface recognition. Several hundred lymphoblastoid cell lines were established by EBV transformation of SLE B cells; monoclonal antibodies were generated by fusion and subcloning. The present study concentrates on two such antibodies, the first (RN86), specific for apoptotic cells and the second (PR5), non-discriminatory in this regard; in both instances, reactivity appeared independent of cell lineage. RN86, whose reactivity was confined to Annexin-V positive cells upon induction of apoptosis, recognized antigen(s) in the $50-70 \mathrm{kDa}$ range. Analysis on a panel of human auto-antigens demonstrated recognition of ribonucleoproteins Ro60, Ro52 and of dsDNA, confirming the polyreactive nature of this antibody. Rat anti-RN86 anti-sera inhibited RN86cell interaction, and Western blot analysis revealed the recognition of antigens distinct from those recognized by RN86. An enhancing influence of RN86 upon the phagocytosis of apoptotic cells by macrophages was observed. PR5, the antibody reactive towards healthy cells, induced the complement-mediated death of cell lines and erythrocytes to varying degrees. Despite its apparent cellular polyreactivity, Western blots demonstrated preferential recognition of a single $30 \mathrm{kDa}$ protein upon erythrocytes; further characterization of this antigen is ongoing. Though sequence analysis of RN86 (Light chain $\mathrm{V} \kappa 1$ (02), J $\kappa 1$; Heavy chain VH3 (V3-53), D3-10, JH6) and PR5 (Light chain V $\kappa$ III (L6), J $\kappa 2$; Heavy chain VH1 (08), D1-26, JH4) revealed very few mutations, extensive non-templated changes occurred at the V-D and D-J junctions. These studies reveal that germline encoded antibodies, present in the early stages of an anti-self response, may contribute to pathogenesis of systemic autoimmunity in several different ways; by inducing the death of healthy cells, by enhancing the phagocytosis of apoptotic cells thereby influencing self-antigen availability and by the diversification of the anti-ribonucleoprotein autoimmune response via the idiotypic network.

\section{[Poster 12]}

Characterization of human hybridoma clones isolated from hemophilia patients with specificity for different domains of coagulating factor VIII Soheila Gharagozlou ${ }^{\mathrm{a}, \mathrm{b}}$, Roya Ghods ${ }^{\mathrm{a}, \mathrm{c}}$, Zahra Samadi Bahrami ${ }^{\mathrm{b}}$, Azam Roohi ${ }^{\mathrm{a}}$, Mahmood Jeddi-Tehrani ${ }^{\text {c,d }}$, Bianca M. Conti-Fine ${ }^{\mathrm{e}}$, Ramazan A. Sharifian $^{\mathrm{f}}$, Hodjatt Rabbani ${ }^{\mathrm{c}, \mathrm{d}}$, Fazel Shokri ${ }^{\mathrm{a}, \mathrm{b}, \mathrm{c}}$

${ }^{a}$ Department of Immunology, School of Public Health, Tehran University of Medical Sciences, Tehran

${ }^{\mathrm{b}}$ National Cell Bank of Iran, Pasteur Inst. of Iran, Tehran

${ }^{\mathrm{c}}$ Monoclonal Antibody Research Center, Avesina Research Center, Tehran

${ }^{\mathrm{d}}$ Immune and Gene Therapy Lab, Cancer Center

Karolinska, Karolinska Hospital, Stockholm

${ }^{\mathrm{e}}$ Department of Biochemistry, Molecular Biology and Biophysics, University of Minnesota, Minneapolis, Minnesota

${ }^{\mathrm{f}}$ Clinic of Hematology and Oncology, Faculty of Medicine, Tehran University of Medical Sciences, Tehran, Iran Tel.: +21 8953021; Fax: +21 6462267;

E-mail:fazshok@yahoo.com 
Introduction: Hemophilia A is a X-linked, hereditary abnormality of blood coagulation, characterized by low or undetectable amounts of coagulation factor VIII (FVIII) in the blood. FVIII replacement therapy is the preferred treatment of the bleeding episodes. However, up to $30 \%$ of severe hemophilia A patients develop anti-FVIII antibodies $(\mathrm{Ab})$ in response to multiple FVIII infusions, which inhibit the pro-coagulant function of FVIII [1]. The human immune response to FVIII is highly heterogeneous. Anti-FVIII Ab may belong to any $\mathrm{IgG}$ isotypes, although $\mathrm{IgG} 4 \mathrm{Ab}$ are involved preferentially [2]. Anti-FVIII Abs may recognize a variety of epitopes: the A2, C2 and to a lesser extent the A3 domains are the most immunogenic parts of FVIII that bind Abs from inhibitor plasmas $[3,4]$.

Elucidation of the molecular mechanisms by which the inhibitors interfere with FVIII function will facilitate the development of a new generation of therapies. In this study, we have established and characterized several different hybridoma clones, obtained from hemophilia A patients. This has provided the opportunity to get insights into the structural and functional features of individual human anti-FVIII Ab.

Materials and methods: Blood collection and peripheral blood mononuclear cell (PBMC) separation: Twenty five milliliter $(\mathrm{ml})$ citrated peripheral blood samples were collected from two hemophilia A patients having high titers of inhibitor in their plasma. PBMCs were prepared by Ficoll density centrifugation [5] using Histopaque (Sigma, USA).

EBV-immortalization of human B-cells: One $\mathrm{ml}$ of filtered supernatant from EBV infected B95.8 marmoset cell line (NCBI C-110; National Cell Bank of Iran, Iran) was added to $10^{6}$ pelleted PBMCs. The cells were seeded at 40000, 80000 and 160000 cells/well in $100 \mu 1$ Dulbeco's Modified Eagle Medium (DMEM) (Sigma) containing 20\% Fetal Calf Serum (FCS) (GIBCO BRL, UK) in 96-well microtitre plates (Griener, Germany) on a feeder layer of fetal lung fibroblasts (MRC-5; NCBI C-125; National Cell Bank of Iran).

Fusion of LCLs with heteromyeloma cells: Wells containing positive LCLs (producing anti-FVIII Abs) were selected, mixed and fused with the mousexhuman heteromyeloma (F3B6) (NCBI C-197; National Cell Bank of Iran) at a ratio of 1:3 using Sterile $50 \% \mathrm{~W} / \mathrm{V}$ polyethylene glycol (PEG 1500) (Sigma) [6]. The resulting cell suspension in DMEM containing $20 \%$ FCS were plated out in 96-well culture plates. On the following day and every 3-4 days thereafter the cells were fed with fresh medium supplemented with HAT (hy- poxantin $1 \times 10^{-4} \mathrm{M}$, aminopterin $4 \times 10^{-7} \mathrm{M}$ and thymidine $\left.16 \times 10^{-6} \mathrm{M}\right)$ and Ouabain $\left(2 \times 10^{-6} \mathrm{M}\right)$ (Sigma).

Screening of anti-FVIII Ab in culture supernatant: After 2-3 weeks of culture, the supernatant of wells containing immortalized B-cells (LCLs) or hybridomas were assayed for production of specific Abs, using an indirect ELISA [7] and positive wells cloned by limiting dilution. culture supernatants were added to the human FVIII coated plates followed by addition of horseradish peroxidase-conjugated rabbit antihuman Ig (HRP-Rb $\alpha$ HIg) (Sigma), the reaction was revealed with O-phenylendiamin dihydrochloride(OPD) substrate (Sigma).

Determination of Ig isotypes and cross-reactive idiotypes (CRIs): An indirect ELISA was employed to determine isotype and CRIs of anti-FVIII Ab in supernatants using isotype specific HRP-Sh $\alpha \mathrm{HIg}$ heavy or light chain (Sigma), monoclonal antibodies specific for human IgG subclasses (kindly provided by Professor Roy Jefferis, Dept. of Immunology, University of Birmingham, UK), and anti-CRI MAbs.

FVIII polypeptide specificity of monoclonal Abs determined by immunoblotting: Approximately $1 \mu \mathrm{g}$ of recombinant FVIII (Helixate FS, Bayer, USA) (thrombin digested or undigested) were loaded onto a $8.5 \%$ polyacrylamid slab gel. Electrophoresed FVIII was transferred on to nitrocellulose membrane (Biorad, USA) and incubated with hybridomas supernatant overnight. HRP-Sh $\alpha \mathrm{HIg}$, IgG, IgM, IgA, kappa and lambda light chains (Sigma) were used to reveal the reaction.

PCR amplification of the $V_{H}$ region genes: Total RNA was extracted from the hybridoma clones using RNAzol B reagent (BioSite, Täby, Sweden) based on the manufacturer instructions. First strand cDNA was synthesized using $5 \mu \mathrm{g}$ of total RNA and M-MLV reverse transcriptase 200 units (GIBCO/BRL, Life Technologies). Thereafter, the mixture was incubated at $42^{\circ} \mathrm{C}$ for $45 \mathrm{~min}$. Serial dilution of cDNA was prepared and PCR amplification was performed using $\mathrm{V}_{\mathrm{H}} 1-6$ primers as sense and $\mathrm{C} \mu$ primer as antisense [8].

Results and discussion: The PBMCs of Two patients (HP1 and HP5) with higher titers of anti-FVIII Ab were infected with EBV and co-cultured at different cell densities. We obtained a total of 15 FVIII-specific LCLs from the two patients (11 from HP1 and 4 from HP5). All LCLs produced IgM and were negative for IgG or IgA. After four rounds of cell fusions, cloning and subcloning we obtained a total of 8 hybridomas producing high titers of anti-FVIII Ab. The reactivity of secreted 
Table 2

Reactivity and isotype profile of anti-FVIII antibody produced by heterohybridoma clones

\begin{tabular}{lcclccc}
\hline Hybridoma clones & *Anti-FVIII titer & \multicolumn{5}{c}{ Isotype (OD) } \\
\cline { 3 - 7 } & OD & IgG & IgA & IgM & Kappa & Lambda \\
\hline F1P3C3 & 2.88 & 0.03 & 0.06 & 1.93 & 2.87 & 0.15 \\
F5P4F8 & 1.25 & 0.01 & 0 & 1.12 & 0 & 2.21 \\
F5P1G11 & 0.98 & 0.01 & 0 & 0.53 & 1.77 & 0 \\
F6P1F11 & 0.76 & 0.06 & 0.02 & 0.41 & 0 & 1.12 \\
F6P5D11 & 1.35 & 0.01 & 0 & 0.82 & 2.17 & 0 \\
F6P4D9 & 0.98 & 0.01 & 0 & 0.27 & 0.84 & 0 \\
F6P2F3 & 1.28 & 0.03 & 0 & 1.3 & 0 & 2.38 \\
F7P1G3 & 1.66 & 0.04 & 0.02 & 1.04 & 2.05 & 0.04 \\
F4.11** & 0.08 & .035 & 0 & 0.01 & 0 & 0.02 \\
\hline
\end{tabular}

*Anti-FVIII titer is presented as OD values obtained by ELISA at $492 \mathrm{~nm}$.

${ }^{* *} \mathrm{~F} 4.11$ is a hybridoma clone producing IgM of unknown specificity.

Abs for FVIII, and the isotypic profile were assessed by ELISA (Table 2). All hybridoma clones were found to secrete anti-FVIII IgM, associated with either a $\kappa$ (5out of $8,63 \%)$ or a $\lambda(3$ out of $8,37 \%$ ) light chain. This isotypic profile differs from that observed in the plasma of hemophilia A patients, where IgG4 inhibitors are the predominant isotype. The exclusive presence of FVIII-specific B-cell clones which express IgM might be explained by an unknown mechanism at the level of FVIII specific memory B-cells, by which circulating IgG producing memory B-cells in the periphery are either silenced or deleted.

Immunoblotting of the FVIII-specific MAbs demonstrated three patterns of reactivity: one included clones reacting exclusively with the $44 \mathrm{kD}$ thrombin-generated A2 domain polypeptide (F6P1F11 and F6P5D11); another included clones reacting with the $72 \mathrm{kD}$ and/or its $80 \mathrm{kD}$ light chain precursor (F5P4F8 and F1P3C3); yet a third pattern was that of MAbs reacting with both the heavy and the light chain polypeptides (F6P2F3 and F7P1G3). Two of the hybridoma supernatants (F5P1G11 and F6P4D9) did not show a clear pattern of reactivity with either digested or undigested FVIII.

According to RT-PCR results five (63\%) of the clones (F1P3C3, F5P4F8, F5P1G11, F6P1F11, F6P2F3) expressed $\mathrm{V}_{\mathrm{H}} 1$, and the other three (F6P5D11, F6P4D9, F7P1G3) expressed the $\mathrm{V}_{\mathrm{H}} 3$ gene families. Among the clones which expressed the $\mathrm{V}_{\mathrm{H}} 1$ gene family, F5P4F8 expressed the $\mathrm{V}_{\mathrm{H}} 1$-associated CRI recognized by the MAb G8. None of the $\mathrm{V}_{\mathrm{H}} 3$ gene family-expressing clones displayed the $\mathrm{V}_{\mathrm{H}} 3$-associated CRI, although two of them (F6P5D11, F7P1G3) coexpressed the epitopes recognized by the $\mathrm{C} 7$ and 17-109 MAbs. The frequency of $\mathrm{V}_{\mathrm{H}}$ gene expression by our hybridoma clones show a predominant use of the $\mathrm{V}_{\mathrm{H}} 1$ gene family (63\%). This is higher than the frequency of functional $\mathrm{V}_{\mathrm{H}} 1$ germline genes $(11 / 51,21.5 \%)$ [9]. The other three hy- bridoma clones (27\%) used the $\mathrm{V}_{\mathrm{H}} 3$ gene family. The human $\mathrm{V}_{\mathrm{H}} 3$ gene family includes the highest number of functional germline $\mathrm{V}_{\mathrm{H}}$ genes $(22 / 51,43.1 \%)$ [9]. Therefore, this gene family is less presented in our hybridoma clones than one would expect from random usage. The over-representation of $\mathrm{V}_{\mathrm{H}} 1$ family, the underrepresentation of the $\mathrm{V}_{\mathrm{H}} 3$ family, and the absence of all the other $\mathrm{V}_{\mathrm{H}}$ gene families, all indicate a biased utilization of the $\mathrm{V}_{\mathrm{H}}$ gene repertoire in shaping the anti-FVIII specificity. Lack of expression of the probed CRIs, and in particular of G6, B6 and D12, may suggest the involvement of somatic mutations in the variable region of our MAbs. Although somatic mutation is usually initiated at the same time as isotype switching in the germinal centers, these two events are mutually exclusive, and extensively mutated IgM molecules have also been reported [10].

\section{References}

[1] L.W. Hoyer, Why do many hemophilia A patients develop an inhibitor? Br J Haematol 90 (1995), 498-501.

[2] J.G. Gilles, J. Arnout, J. Vermylen and J.M.R. Saint-remy, Anti-factor VIII antibodies of haemophiliac patients are frequently directed towards nonfunctional determinants and do not exhibit isotypic restriction, Blood 82 (1993), 2452-2461.

[3] C. Fulcher, S. De Graaf Mahoney, J. Roberts, C. Kaspe and T. Zimmerman, Localization of human FVIII inhibitor epitopes to two polypeptide fragments, Proc Natl Acad Sci USA 82 (1985), 7728-7732.

[4] D. Scandella, S. De Graaf Mahoney, M. Mattingly, D. Roeder, L. Timmons and C. Fulcher, Epitope mapping of human FVIII inhibitor antibodies by deletion analysis of FVIII fragments expressed in Escherichia Coli, Proc Natl Acad Sci USA 85 (1988), 6152-6156.

[5] F. Shokri, R.A. Mageed, B.R. Maziak and R. Jefferis, Expression of VHIII-associated cross-reactive idiotype on human B-lymphocytes: Association with staphylococcal protein A (SPA) binding and staphylococcus aureus Cowan I (SAC) stimulation, J Immunol 146 (1991), 936-940. 
[6] R. Payam khaja Pasha, A. Roohi and F. Shokri, Establishment of heterohybridoma and lymphoblastoid cell lines specific for the Rh D and C antigens, Transfusion Medicine 13 (2003), 83-92.

[7] F. Shokri, R.A. Mageed and R. Jefferis, A quantitative ELISA for measurement of RF-associated CRI in serum from patients with rheumatic disease, Br J Rheum 32 (1993), 862-869.

[8] E.W. Schettino, S.K. Chai, M.T. Kasain, H.W. Schroeder and P. Jr Casali, VHDJH gene sequences and antigen reactivity of monoclonal antibodies produced by human B-1 cells: evidence for somatic selection, J Immunol 158 (1997), 24772489.

[9] E. Vargaz-Madrazo, F. Lara-Ochoa and M.C. RamirezBenites, Evolution of the structural repertoire of the human VH and VK germline genes, Int Immunol 9 (1997), 1801-1815.

[10] H.P. Brezinschek, S.J. Foster, R.I. Brezinschek, T. Dorner, R. Domiati-Saad and P.E. Lipsky, Analysis of the human $V_{H}$ gene repertoire: Differential effects of selection and somatic hypermutation on human peripheral $\mathrm{CD}^{+} / \mathrm{IgM}^{+}$and $\mathrm{CD} 5^{-} / \mathrm{IgM}^{+}$ B cells, J Clin Invest 99 (1997), 2488-2501.

[Poster 13]

Human monoclonal antibodies to carcinoembryonic antigen (CEA) generated by using the KM mouse ${ }^{\mathrm{TM}}$

Hirotomo Shibaguchi $^{\text {a }}$, Takayuki Imakiire ${ }^{\mathrm{a}}$, Motomu Kuroki $^{\mathrm{a}}$, Adel Badran ${ }^{\mathrm{b}}$, Jitian Zhang ${ }^{\mathrm{b}, \mathrm{c}}$, Ken Hachimine $^{\mathrm{a}, \mathrm{c}}$, Takayuki Shirakusa ${ }^{\mathrm{c}}$, Isao Ishida ${ }^{\mathrm{d}}$ and Masahide Kuroki ${ }^{\mathrm{a}, \mathrm{b}}$

a Department of Biochemistry, Fukuoka University School of Medicine, Fukuoka 814-0180, Japan ${ }^{\mathrm{b}}$ Molecular Oncology Center, Fukuoka University School of Medicine, Fukuoka 814-0180, Japan ' Second Department of Surgery, Fukuoka University School of Medicine, Fukuoka 814-0180, Japan

${ }^{\mathrm{d}}$ Central Laboratory for Key Technology, Kirin Brewery Co. Ltd., Yokohama 236-0004, Japan

We generated fully human mAbs (HmAbs) to carcinoembryonic antigen (CEA) using the KM mouse ${ }^{\mathrm{TM}}$, which carries a human chromosome 14 fragment containing the entire Ig $\mathrm{H}$ chain loci and human? L chain segments in the mouse genome. Forty-six hybridoma clones producing $\mathrm{HmAbs}$ to CEA were thus obtained by fusing the P3-U1 mouse myeloma cells with splenocytes of the KM mice immunized with CEA. Among them, 22 clones produced HmAbs that reacted with CEA but not with three other CEA-related cell adhesion molecule (CEACAM) family members, CEACAM1, CEACAM6 and CEACAM8. In 12 HmAbs examined, eight were $\operatorname{IgG} 4$, two were $\mathrm{IgG} 3$, one was $\mathrm{IgG} 2$, and the other was IgG1. The affinity constants for CEA of these HmAbs were comparable to those of the previously prepared mouse anti-CEA mAbs (MmAbs). BIAcore analyses revealed that one and two of the 22
HmAbs react with two epitopes defined by MmAbs on the domain $\mathrm{N}$ and the domain $\mathrm{A} 1$ or $\mathrm{B} 1$ of CEA, respectively. In the presence of human complement in vitro, two HmAbs tested showed substantial cytotoxicity, namely 50 to $65 \%$, against CEA-expressing tumor cells. With human lymphokine-activated killer cells in vitro, three HmAbs tested exhibited 40 to $65 \%$ Abdependent cell-mediated cytotoxicity against the tumor cells. Moreover, one of the HmAbs induced a significant inhibition of tumor growth when administered to mice xenografted with the CEA-expressing cells. Considering their lack of immunogenicity to humans, these CEA-specific HmAbs may be useful for immunotherapeutic approaches as well as for immunodiagnosis.

\section{[Poster 14]}

Isolation and sequence analysis of anti-oxidized LDL antigen-binding fragments from atherosclerosis patients generated by phage display Eun Sun Yu ${ }^{\mathrm{a}}$, Eun-Young Kang ${ }^{\mathrm{a}}$, Byung-il W. Choi ${ }^{\mathrm{b}}$, Hyuk-Jae Chang ${ }^{\mathrm{a}}$ and Young-Ju Jang ${ }^{\mathrm{a}}$,*

${ }^{\mathrm{a}}$ Laboratory of Immunology, Institute for Medical Science, Ajou University School of Medicine, Suwon, 442-721, Korea

${ }^{\mathrm{b}}$ Department of Cardiology, Ajou University School of Medicine, Suwon, 442-721, Korea

*Corresponding author. E-mail: jangyj@ajou.ac.kr

Oxidative modification of LDL plays a significant role in experimental atherosclerosis and the oxidized LDL may represent a target for autoantibodies involved in the pathogenesis of the disease. To investigate the origin and mechanism of the pathogenic antioxLDL autoantibody production, we isolated ten antioxLDL monoclonal Fabs from atherosclerosis patients and healthy individuals using phage display technology and analyzed their molecular features. The heavy (H) and light (L) chain V-region sequences of the Fabs (P2-8, P2-32, P2-114, P2-147, P3-116, P3-155, P3175, P3-190, H1-132, H2-38 and H2-119) were determined at the cDNA level. Only two different VH families were represented; four of the patient' Fabs used $\mathrm{V}_{\mathrm{H}} 3$, three of them used $\mathrm{V}_{\mathrm{H}} 1$ gene family. All three Fabs from healthy individuals used $\mathrm{V}_{\mathrm{H}} 3$ family. In the case of $\mathrm{V}_{\mathrm{L}}$, diverse gene family elements were represented; V $\kappa 3, \mathrm{~L} 1, \mathrm{~L} 2$ and L3 from clones of patients, $\mathrm{V} ? 3$ being the most common as three of the Fabs. V $\kappa 1$ gene family was present only in two healthy individual's clones. Comparison of the gene segments utilized by these Fabs with their germline counterparts revealed that most of them were mutated extensively; identities 
of their $\mathrm{VH}$ to germline genes were in the range of $88-99 \%$, those of VL were between 87 and $98 \%$. In the clones from both groups, patients and healthy individuals, common features described below were found; the lengths of $\mathrm{H}-\mathrm{CDR} 3$ were highly heterogeneous (520 nucleotides); the R/S mutation ratio in CDRs was tend to be higher than that in FRs of both $\mathrm{V}_{\mathrm{H}}$ and $\mathrm{V}_{\mathrm{L}}$; aromatic amino acids, Tyr, Phe and Trp, occurred frequently in $\mathrm{H}-\mathrm{CDR} 3$ of all clones; in the $\mathrm{V}_{\mathrm{L}}$ regions, high frequencies of Phe and His mutations were observed. Only a distinct property of Fab clones between two groups was the presence of Ser residue in L-CDR3 of all patient's clones, not healthy individual's, and it may be important for conferring pathogenic property of anti-oxLDL autoantibodies. These results may suggest that an antigen-driven process may occur during the selection of the natural or pathogenic anti-oxLDL autoantibody-producing B cells, and acquisition of certain amino acids, such as Ser residue, in L-CDR3 might effect on the pathogenicity of the antibodies.
[Poster 15]

Molecular charcetrization and expression of neutralizing human antibodies specific for the Entamoeba histolytica Gal/GalNAc lectin heavy subunit

H. Tachibana et al.

Tokai University School of Medicine, Kanegawa, Japan

Abstract not received.

[Poster 16]

Development of a potential therapeutic antibody against respiratory syncytial virus

C. Mekseepralard

Srinikharinwirot University, Bangkok, Thailand

Abstract not received. 